those with relatively low radon exposure. For any given level of radon, smokers had about 25 times the absolute risk of developing lung cancer of non-smokers.

The authors conclude that radon in the home poses a significant hazard, particularly among smokers, and is to blame for around $2 \%$ of all cancer deaths in Europe.

Original article Darby S et al. (2004) Radon in homes and risk of lung cancer: collaborative analysis of individual data from 13 European case-control studies. BMJ [doi: 10.1136/ bmj.38308.477650.63]

\section{Dietary magnesium and colorectal cancer risk}

A large, prospective study from Sweden has suggested that a high intake of magnesium lowers the risk of colorectal cancer in women. These findings reflect experimental studies suggesting that magnesium may inhibit colorectal carcinogenesis.

Larsson et al. used questionnaire data provided by 61,433 women from the Swedish Mammography Cohort, which was established between 1987 and 1990. Using the Swedish National Food Administration Database, the investigators estimated the energy-adjusted daily magnesium intake of each participant and then categorized the women into quintiles on this basis. During a mean follow-up of 14.8 years, a statistically significant, inverse relationship was observed between magnesium intake and colorectal cancer risk $(P=0.006)$. Women in the highest quintile of magnesium intake had a lower risk of colorectal cancer than those in the lowest quintile (multivariate rate ratio 0.59, 95\% Cl 0.40-0.87), and a similar pattern was observed when colon cancer and rectal cancer were analyzed separately.

These results support the idea that increasing consumption of foods that contain magnesium - such as fruit and vegetables, whole grain foods and beans-can reduce the risk of colorectal cancer. The authors note, however, that randomized studies will be needed to examine the safety and potential benefits of magnesium supplementation.

Original article Larsson SC et al. (2005) Magnesium intake in relation to risk of colorectal cancer in women. JAMA 293: 86-89

\section{Is total androgen blockade useful in metastatic prostate cancer?}

A recent study by Ansari and colleagues has shown that orchiectomy alone was equally as effective as orchiectomy plus flutamide in the treatment of men with metastatic carcinoma of the prostate.

The study included 100 patients with advanced prostate cancer who were randomized in a 1:1 ratio to orchiectomy alone $(0)$ or orchiectomy plus flutamide (OF), and were followed up for a mean period of 3.5 years. The maximum percentage decrease in serum PSA levels was similar in both groups $(95 \%$ and $97 \%$ for groups $\mathrm{O}$ and OF, respectively), and occurred during the first 3 months after orchiectomy. This decrease in PSA persisted for 3 years in more than $80 \%$ of patients in both groups. There was no significant difference between the $\mathrm{O}$ and OF groups in the proportion of patients experiencing complete or partial response, as indicated by serum PSA levels and bone scan findings. The mean time to progression was 27 and 29 months in the $O$ and OF groups, respectively, and overall survival was similar in the two groups at 3 years $(45.8 \%$ vs $48.1 \%)$ and 5 years (20.8\% vs $23.1 \%)$.

In summary, total androgen blockade was not superior to orchiectomy alone in decreasing serum PSA in this study, and overall survival was similar using the two strategies. The authors, therefore, advise against the routine addition of flutamide in patients undergoing orchiectomy.

Original article Ansari MS et al. (2004) Combined androgen blockade in the management of advanced prostate cancer: a sensible or ostensible approach. Int J Urol 11: 1092-1096

\section{Prostate cancer treatment and osteoporosis risk}

Insufficient attention is given to the risk of osteoporosis in men receiving androgen deprivation therapy (ADT), according to a recent study published in Cancer. Although ADT is a known risk factor for osteoporosis, few patients appear to receive the appropriate interventions for the prevention or treatment of the disease.

Tawee Tanvetyanon carried out a retrospective analysis of 184 men with prostate cancer, all of 